\title{
Multi-electron Bubbles under Pressure
}

\author{
ISAAC F. SILVERA ${ }^{1}$, JACQUES TEMPERE ${ }^{1,2}$, JIAN HUANG ${ }^{1}$, AND \\ JOZEF DEVREESE ${ }^{2}$ \\ ${ }^{1}$ Lyman Laboratory of Physics, Harvard University, Cambridge MA, 02138 \\ ${ }^{2}$ Dept. Natuurkunde, Universiteit Antwerpen UIA, B2610 Antwerpen, Belgium
}

\begin{abstract}
Multi-electron bubbles (MEBs) can be macroscopic in size. The crucial surface electron density is limited to a bit more than $10^{9} \mathrm{~cm}^{-2}$ on flat helium surfaces, but can be greater than $10^{12} \mathrm{~cm}^{-2}$ in small bubbles and as high as $10^{14} \mathrm{~cm}^{-2}$ for helium under modest pressures and can have increased stability for negative pressures. We discuss some of the properties of as well as a method for stabilizing of MEBs.
\end{abstract}

Key Words : Electrons, helium, pressure, Wigner crystallization.

\section{INTRODUCTION}

The multi-electron bubble (MEB) is essentially a spherical bubble containing from 2 to more than $10^{8}$ electrons inside of liquid helium. A single electron bubble (SEB) has a radius of $34 \AA$ [1] while, for example, a bubble of $10^{4}$ electrons theoretically should have a radius of around one micron. The surprisingly large diameter of the SEB is the result of a balance between the zero-point energy of the electron and the surface tension energy of the liquid helium. A spherical surface has the minimum energy (in the absence of gravity) and is thus the expected shape of a static bubble or a bubble in its ground state. For larger bubbles electrons repel each other due to Coulomb forces and reside in a thin shell of order 10-20 $\AA$ wide on the inner surface of the bubble, and at about the same distance from the He surface. For small 
bubbles with only a few electrons, the zero-point energy is important and the electrons have some distribution throughout the volume. We shall focus our attention on larger bubbles. The electrons form a well-defined twodimensional electron gas (2DEG) on a spherical surface. Larger bubbles can be deformed from the spherical shape in a gravitational field, as well sessile bubbles, but here we shall be concerned with spherical bubbles.

Although high pressure techniques have been developed to reach static pressure as high as $5 \times 10^{6}$ bars, the importance of pressure to the physics of the problem is the compression of the matter, not the pressure. The combination of helium and the $2 \mathrm{DEG}$ are very compressible and respond to both positive and negative pressures with enormous compression that will be discussed. In the case of MEBs significant effects are observed for pressures less than 1 bar, both positive and negative. Since the electron surface density is crucial for the physics of bubbles, later we shall discuss the areal compression, rather than the volume compression.

The observation of an MEB was a result of studies of 2DEGs on flat helium surfaces. An electron is attracted to a liquid helium surface by its image charge, forming a Coulomb attractive potential in the direction perpendicular to the surface. There is a barrier of about $1 \mathrm{eV}$ for an electron to penetrate into the helium [2], so that the electron falls into a bound state on the surface. Early studies a few decades ago were motivated by a desire to observe Wigner crystallization [3] of the 2DEG into a triangular lattice of electrons. Since the helium surface charges up with electrons, in order to increase the surface density, a positively charged electrode was placed under the helium surface. The highest densities observed in this configuration were a few times $10 \% / \mathrm{cm}^{2}$. At higher densities an instability develops in which the helium surface deforms to subsume large numbers of electrons (millions) in an MEB. This MEB is short-lived as it moves with a fairly high mobility through the helium to the electrode, where it is annihilated. These bubbles have been photographed [4] and their mobility measured by optical techniques [5], but there have been no detailed studies of their properties due to their short lifetimes, of order milliseconds. Although there are some theoretical questions concerning the stability of an MEB (against fissioning into smaller bubbles) all observations indicate that they are stable, but short-lived due to the method of production. There have been a few proposals in the literature for techniques to stabilize MEBs, but none have been successfully executed [6-7]. In this paper we shall discuss some of the properties and a new proposal for the stabilization of MEBs [8]. 


\section{PROPERTIES OF MEBs}

It is useful to first develop some properties of the static MEB, such as the relationship between the number of electrons $\mathrm{N}$, and the radius, called the Coulomb radius, $R_{c}$, found by minimizing the static energy of the bubble,

$$
\begin{aligned}
U= & 4 \pi \sigma R_{b}^{2}+\frac{e^{2} N^{2}}{2 \varepsilon\left(R_{b}-d\right)}+\frac{N \hbar^{2}}{2 m_{e} d^{2}}-0.3176 \frac{e^{2} N^{4 / 3}}{\varepsilon\left(R_{b}^{2} d\right)^{1 / 3}} \\
& +\frac{4 \pi}{3} p R_{b}^{3} .
\end{aligned}
$$

Here the first term is the energy due to the surface tension of the helium, with $\sigma$ the surface tension; the second term is the Coulomb repulsion, with $\varepsilon$ the dielectric constant of helium, and $d$ the distance of the electron layer from the surface, as suggested by Shikin [9]. The third term is the localization zero-point confinement energy. The fourth term is the exchange energy after the density functional calculations of Shung and Lin [10]. The third and fourth terms are important for small bubbles with $N \leq 10^{3}$. The final term is the hydrostatic pressure, $\mathrm{p}$, applied to the liquid helium. A static bubble with $\mathrm{p}=0$ is apparently stable, but from Eq. (1) it is easy to see that the energy scales as $\mathrm{N}^{4}$ (ignoring terms important for small bubbles). It is clear that a bubble of $N$ electrons has a higher energy than two with $N / 2$ electrons each, so there evidently is a formation barrier that stabilizes single bubbles. A study of the free-energy shows that an MEB is stable against radial deformations; however angular deformations may lead to instabilities, as we shall see.

Taking $\mathrm{p}=0$ in Eq. (1) and minimizing, we find for large bubbles

$$
R_{c}=\left[\frac{e^{2} N^{2}}{16 \pi \sigma \varepsilon}\right]^{1 / 3}=1.064\left(\frac{N}{10^{4}}\right)^{2 / 3} \text { microns }
$$

From this the surface density of the 2DEG easily follows; dividing $\mathrm{N}$ by surface area of a bubble yields 


$$
\begin{aligned}
n_{s} & =N^{-1 / 3}\left(16 \pi \varepsilon \sigma / e^{2}\right)^{2 / 3} \\
& =7.025 \times 10^{10}\left(\frac{N}{10^{4}}\right)^{-1 / 3} \mathrm{~cm}^{-2} .
\end{aligned}
$$

We see that for fixed pressure, smaller bubbles have higher electron surface density. In Fig. 1 we show in a three-dimensional plot the surface density vs. $N$ and pressure. An enormous range is spanned, with density orders of magnitude greater than achievable on flat surfaces. Changing the number of electrons in the MEB at constant pressure varies these densities. An alternate method that further expands the density range is to apply pressure to the helium, for a fixed number of electrons up to pressures of 25 bar, roughly where liquid helium solidifies. Liquid helium has a "tensile" strength and can sustain negative pressures of 9 bars before it cavitates [11-12]. However, if a bubble is included within the helium, the bubble will expand as the absolute value of the negative pressure increases, until the bubble becomes unstable and expands indefinitely [13]. The critical negative pressures are in the millibar range, depending on the number of electrons in the bubble. Negative pressure (not plotted in Fig. 1) can expand the bubble radius up to 1.5 times its zero pressure value, decreasing the lowest achievable density by a further factor of 0.44 .

The MEB is expected to have fascinating properties and we shall discuss a few of these below. Consider first the low-lying excitations of the system. These are deformations of the surface of the bubble in which the electron gas conforms to the helium surface; these modes are called spherical ripplons and are closely related to ripples or surface waves on a flat surface. Ripplons can be quantized and the wave functions are spherical harmonics; the harmonic modes are labeled by quantum numbers $\ell$ and $\mathrm{m}$, the latter being degenerate. Oscillation frequencies are in the acoustic to radio frequency range. 


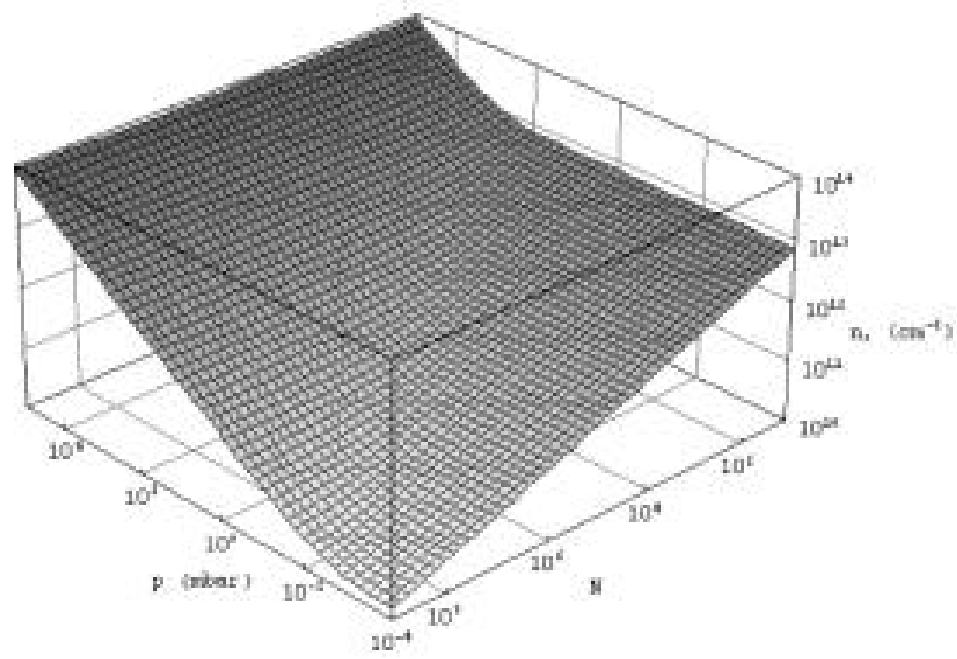

Fig. 1: The surface density as a function of both the number of electrons in the bubble and the pressure. All axes are logarithmic. The density of electrons exceeds the typical $10^{9} \mathrm{~cm}^{-2}$ on a flat helium surface, and can be varied easily in the range $10^{10}-10^{14} \mathrm{~cm}^{-2}$.

By developing the kinetic energy function and the Lagarangian for the angular deformations of the bubble, the frequencies and corresponding deformation amplitudes $Q_{t m}$ of these modes can be calculated [13]. The resulting mode frequencies (in the limit of large bubbles, $N \geq 10^{3}$ ) are

$$
\omega_{\ell}=\sqrt{\frac{\ell^{2}-1}{\rho R_{e q}^{3}}\left[\sigma(\ell-2)-2 p R_{e q}\right]} .
$$

Here $\rho$ is the density of liquid helium, and $R_{e q}$ is the equilibrium radius of the bubble. For $p=0$, and $R_{e q}=R_{c}$ we see that the $\ell=1$ and 2 modes are soft, i.e., zero frequency. The $\ell=1$ mode corresponds to uniform translation of the bubble, while the $\ell=2$ quadrupole mode represents an instability for fissioning of the bubble. For non-zero negative pressure this instability is lifted while positive pressures will sequentially drive all modes unstable. Ripplon frequencies as a function of pressure are plotted in Fig. 2. In Fig. 3 we show the deformation amplitude for the $\ell, m=7,5$ mode. In this case the amplitudes are greatly exaggerated, whereas in reality the theory is only valid for small amplitude deformations. 


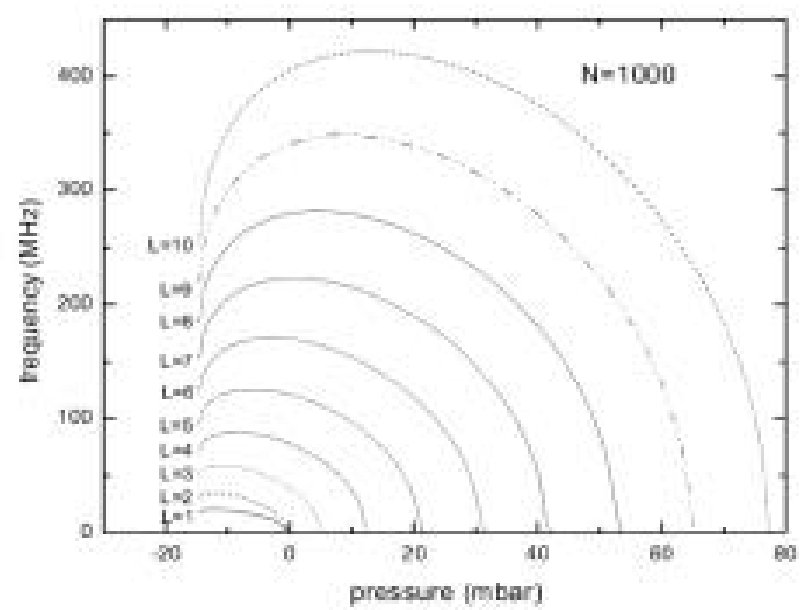

Fig. 2. Ripplon frequencies of MEBs as a function of pressure for a bubble with $\mathrm{N}=1000$, showing how negative pressure stabilizes the $\mathrm{L}=2$ mode and positive pressures destabilize all modes.

Experimental excitation and observation of these modes will be fundamental to the study of the MEBs. For example for a fixed $N$ the frequency should shift depending on if the electrons are a $2 \mathrm{DEG}$ or form a Wigner crystal [14].

Wigner crystallization occurs for a $2 \mathrm{DEG}$ in a flat geometry if the plasma parameter

$$
\Gamma=V_{p o t} / \operatorname{Kin} \geq 137 \pm 15
$$

For so-called classical solidification $V_{p o t}=e^{2}\{1 / r\rangle\left(1 / 4 \pi \varepsilon_{0}\right)$ $=e^{2} \sqrt{\pi n_{s}} / 4 \pi \varepsilon_{0}$, where $r$ is the average distance between electrons, and $\operatorname{Kin}=k_{B} T$. For a flat surface, the lowest energy structure for the Wigner crystal is the triangular lattice. On a spherical surface, although the triangular 


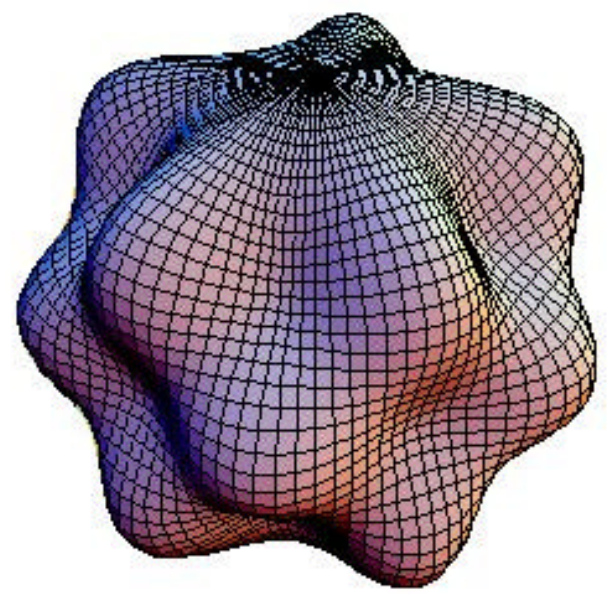

Fig. 3. Example of a deformed bubble - the deformation corresponds to a ripplon mode $\ell, m=7,5$. The amplitude of the deformation is large for clarity of viewing.

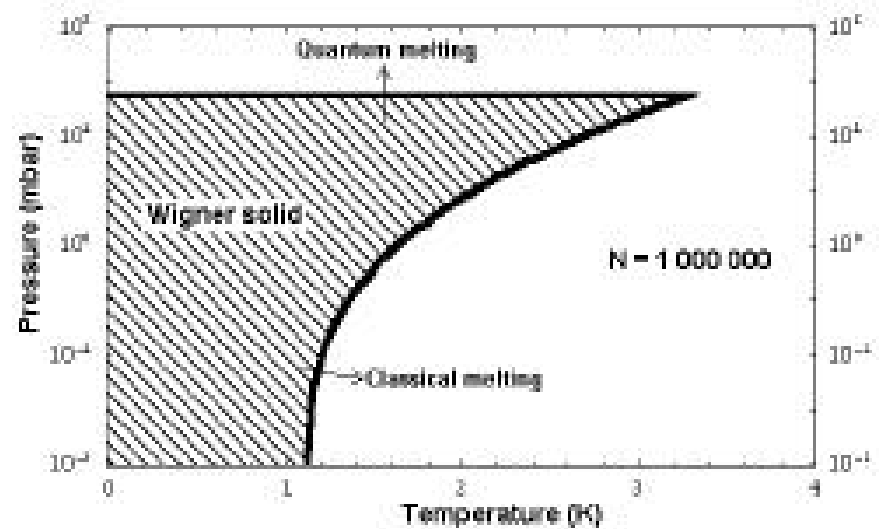

Fig. 4: The region in the temperature-pressure phase diagram for an MEB with one million electrons. When $\Gamma>137$ one might expect Wigner crystallization to occur. Below a threshold pressure, the Wigner solid can be melted by increasing the temperature. Above a threshold pressure, the density of electrons or the zero-point energy is large enough to induce quantum melting of the Wigner solid.

the lattice may be the local lowest energy structure, a triangular net does not fit on surface and defects with an electron having either 5 neighbors or 
neighbors will be built into the ground state of the triangular lattice [14]. Wigner crystallization was observed in the range of $n_{s}=10^{9} \mathrm{~cm}^{-2}$ and $T \approx 1 \mathrm{~K}$ [15] . This is called classical crystallization because the kinetic energy is thermal. At high enough electron surface density a quantum transition is predicted for the Wigner lattice to melting. If the Fermi energy

$E_{f}=\pi \hbar^{2} n_{s} / m>k_{B} T$, then the zero-point energy is $\operatorname{Kin}=E_{f}$; we see that with increasing density, Kin increases faster than $V_{p o t}$ until $\Gamma$ attains the value given by Eq. (5) and the lattice melts. In Fig. 4 we show the phase lines for classical and quantum melting of the Wigner lattice in an electron bubble.

It is interesting to note that the attractive electron-ripplon interaction may lead to superconductivity of the electron gas. The fascinating possibility of superfluid rotation of the Wigner lattice exists. If the 2DEG forms a Wigner solid, can this rotate within the bubble without dissipation? We believe that many of these problems will be accessible if the MEB can be stabilized in a convenient geometry for study.

\section{A PROPOSAL FOR STABILIZATION AND STUDY OF MEBs}

We now discuss an experimental proposal for stabilizing and studying MEBs [8]. In Fig 5 we show schematically an experimental cell. The cylindrical cell has a dome-shaped roof; the bottom is closed by a flexible edge-welded bellows. The cell is partially filled with liquid helium via a capillary that can be sealed at low temperature. Either an electrode or a field emission tip performs as a source of electrons in the volume above the superfluid liquid helium. The helium has a barrier of $1 \mathrm{eV}$ for an electron to penetrate and a helium film covers all surfaces. Electrons created in this volume will either adsorb on the helium surface or be in the 3-D volume. The bellows can be compressed to raise the surface of the helium to the roof of the cell with a nominal value of zero-pressure in the helium. At this position the electrons will be corralled into the dome and will form a minimum energy surface, or an MEB. Further compressing of the bellows will increase the pressure, while expansion (with the fill capillary sealed) develops a negative pressure. The roof of the cell contains a sealed concave glass window that permits viewing of the MEB from above. This window has a transparent 


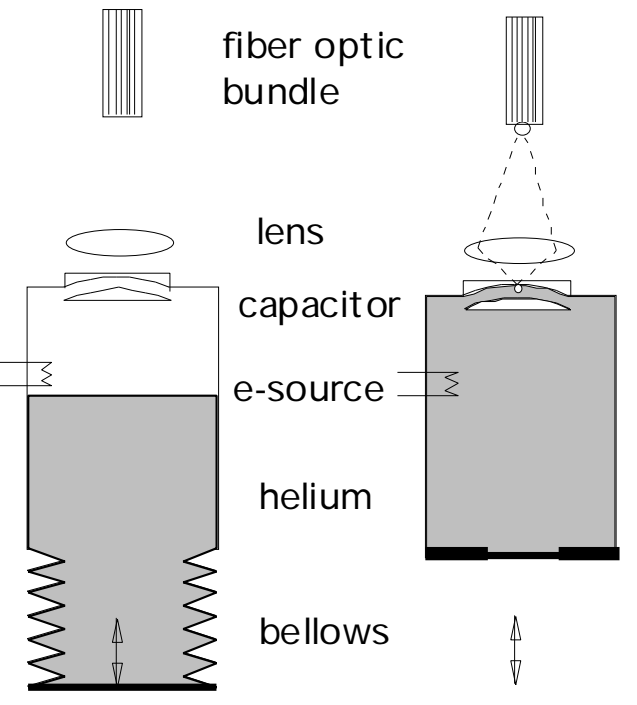

Fig.5. A schematic cell for stabilizing and studying multi-electron bubbles. The fill capillary and valve, as well as a fiber optic illuminator are not shown.

conducting electrode on its surface; a second mating window forms a capacitor that enables the measurement of the charge in the bubble. The bubble can be illuminated with a fiber optic internal to the cell (not shown) and viewed with a coherent fiber optic bundle. Using such a geometry, the static and dynamic properties of MEBs can be studied.

\section{ACKNOWLEDGEMENTS}

Discussions with J.N. Klimin and V. Fomin are gratefully acknowledged. This research was supported by the Department of Energy, grant DE-FG002-85ER45190; the NSF, grant DMR-0071828; GOA BOF UA 2000, IUAP, FWO-V project Nos. G.0071.98, G.0306.00, G.0274.01, WOG 
WO.025.99N (Belgium), and the ESF program VORTEX. J .Tempere is supported financially by the FWO-Flanders.

\section{REFERENCES}

1. C.C. Grimes and G. Adams, Phys. Rev. B 41, 6366 (1990).

2. M.W. Cole, Rev. Mod. Phys. 46, 451 (1974).

3. E. Andrei, Two-Dimensional Electron Systems on Helium and other Cryogenic Substrates. 1997, Dordrecht: Kluwer Acad. Publ.

4. A.P. Volodin, M.S. Khaikin, and V.S. Edel'man, JETP Lett. 26, 543 (1977).

5. U. Albrecht and P. Leiderer, Europhys. Lett. 3, 705 (1987).

6. P. Leiderer, private communication.

7. G.A. Williams, Zeits. fur Phys. B 98, 341 (1995).

8. I.F. Silvera, Bull. Am. Phys. Soc. 46, 1016 (2001).

9. V.B. Shikin, JETP Lett. 27, 39 (1978).

10. K.W.-K. Shung and F.L. Lin, Phys. Rev. B 45, 7491 (1992).

11. H.J. Maris and Q. Xiong, Phys. Rev. Lett. 63, 1078 (1989).

12. I.F. Silvera, J.D. Gillaspy, and J.G. Brisson. Spin Polarized Quantum Systems. 1989. Torino: World Scientific.

13. J. Tempere, I.F. Silvera, and J.T. Devreese, submitted for publication (2001).

14. P. Lenz and D.R. Neson, cond-mat/01040-62 (2001).

15. C.C. Grimes and G. Adams, Phys. Rev. Lett. 42, 795 (1979). 